\title{
MATERIALS CONFERENCES
}

\section{A Complete Calendar Of Every Major Symposium \\ And Technical Conference For Materials Professionals}

For the first time, notices of all major technical meetings of interest to the materials community have been brought together into a single, comprehensive calendar by the Materials Research Society. Prepared by Julia Phillips of Bell Laboratories, the calendar will be continuously updated and will appear in future issues of the BULLETIN.

MARCH 1984

3-5 Short Course on Chemical Lasers

Las Cruces, NM

Engineering Tech., PO Box 8859, Waco, TX 76714 (817) $772-0082$

4-6

Annual Meeting, Canadian Ceramic Society

Toronto, Canada

Canadian Ceramic Society, 2175 Sheppard Av. E, Suite 110

Willowdale, Ontario, M2J 1 W8, Canada

5-6 Short Course on Laser Technology and Systems Applications Atlanta, GA

E. Nicholas, Dept. of Continuing Ed., Georgia Inst. of Tech.

Atlanta, GA 30332

(404) $894-2547$

Short Course on Infrared Technology and Applications Atlanta, GA

E. Nicholas, Dept. of Continuing Ed., Georgia Inst. of Tech.

Atlanta, GA 30332

(404) $894-2547$

11-14 AIChE Winter National Meeting

Atlanta, GA

AIChE, 345 E. 47 St., New York, NY 10017

(212) $705-7320$

19-22 Materials Testing Exhibition - MT '84

Birmingham, UK

Mack-Brooks Exhibitions Ltd., 62 Victoria St., St. Albans,

ALI 3XT, UK

19-23 4th General Conference of the Condensed Matter Division of the European Physical Society

The Hague, Holland

F. M. Mueller, Phys. Lab., Toernooiveld, NL-6525 ED

Nigmegen,

The Netherlands

22-24 Conference on Defects in Amorphous Materials

Detroit, MI

H. Fritasche, James Franck Institute, Univ. of Chicago,

5640 Ellis Av., Chicago, IL 60637

26-30 American Physical Society General Meeting

Detroit, MI

W. W. Havens, Jr., 335 E 45 St., New York, NY 10017

(212) $661-9404$
APRIL 1984

1-4 Vacuum '84: Technological Aspects of Surface Treatment \& Analysis

University of York, UK

J. S. Colligon, Dept. of Electronic \& Electrical

Engineering,

University of Salford, Salford M5 4WT, UK

1-5 6th European Converence on Surface Science

York, UK

Meetings Office, IOP, 47 Belgrave Sq., London SWIX 8QX, UK

6-8 American Vacuum Society Thin Film Division Top Symposium on Sputtering

San Diego, CA

W. D. Westwood, Bell-Northern Research, Box 3511, Station C, Ottawa, Ontario, Canada KIY $4 \mathrm{H} 7$

(613) 596-3248

8-13 187th National Meeting of the American Chemical Society St. Louis, MO

Am. Chem. Soc., Dept. of Meetings, 1155 16th St., NW. Washington, DC 20036

9-11 Topical Meeting on High Temperature Alloys: Theory and Design

Bethesda, MD

C. J. McHargue, Metals \& Ceramics Div., Oak Ridge Natl. Lab.,

P.O. Box X, Oak Ridge, TN 37830

9-12 3rd International Conference on Mechanical Properties of Materials at High Rates of Strain

Oxford, UK

J. Harding, Dept. of Eng. Sci., Parks Rd., Oxford OXI 3PJ, UK

9-13 4th International Symposium on the Properties and Applications of Metal Hydrides

Eliat, Israel

D. Shaltiel, Racah Inst. of Phys., Hebrew Univ. Jerusalem 91904, Israel

11-14 British Ceramic Society Meeting on the Chemistry \& Chemically Related Properties of Cement

London, England

17-20 Optical Society of America Spring Conference Monterey, CA

OSA, 1816 Jefferson Pl., NW, Washington, DC 20036

17-18 Spring '84 Topical Meeting on Science of Polishing Monterey, CA

OSA, 1816 Jefferson Pl., NW, Washington, DC 20036 (202) $223-8130$

23-26 American Physical Society Spring Meeting Washington, DC

W. Havens, Jr., 335 E. 45 St., New York, NY 10017

24-26 3rd International Conference on III-V Semi-Insulating Materials Warm Springs, OR

W. Ford, Harris Semiconductor, 1530 McCarthy Blvd., Milpitas, CA 95035 
EEE 1984 National Symposium on Electromagnetic

Compatibility

San Antonio, TX

W. McGinnis, Southwest Res. Inst., PO Drawer 28510 ,

San Antonio, TX 78284

29-2 86th Annual Meeting and Exposition of the American Ceramic Society

Pittsburgh, PA

S. Robb, American Ceramic Society, 65 Ceramic Dr., Columbus, $\mathrm{OH} 43214$

(614) 268-8645

MAY 1984

7-9 Electroceramics Conference

Brussels, Belgium

9-11 Symposium on Reliability in Ceramic Production \& Utilization Limoges, France

10-11 Composite Materials Conference

Konstanz, West Germany

24-29 AAAS Annual Meeting

New York, NY

AAAS, 1776 Massachusetts Av., NW, Washington, DC 20036

\section{JUNE 1984}

$3-6$

Inter-Disciplinary Workshop on Structure and Properties of the Amorphous State

Williamsburg, VA

J. Voge, Div. of Polymer Chem., Polytechnic Inst. of New York, 333 Jay St., Brooklyn, NY 11201

4-5 Short Course on Techniques for Surface Analysis Dayton, $\mathrm{OH}$

J. T. Grant, Research Inst., Univ. of Dayton, Dayton, OH 45469

5-7 MRS-Europe Technical Conference

Strasbourg, France

6-8 6th Symposium on Applied Surface Analysis

Dayton, $\mathrm{OH}$

10-13 58th Colloid \& Surface Science Symposium 13-17 Pittsburgh, PA

G. D. Parfitt, Chem. Eng. Dept., Carnegie-Mellon Univ.,

Pittsburgh, PA 15213

(412) $578-3848$

18-20 1984 Physical Electronics Conference

Princeton, NJ

J. E. Rowe, Physics Dept., 215 Williamson Hall, Univ. of Florida,

Gainesville, FL

\section{JULY 1984}

2-4 2nd International Symposium on Applications of Laser

Anemometry to Fluid Mechanics

Lisbon, Portugal

D. F. G. Durao, Dept. of Mech. Eng., Inst. Superior Tecnico,

Ave. Rovisco Pais, 1096 Lisbon, Portugal

3-6 International Conference on Crystal Growth and Characterization of Polytype Structures

Marseilles, France

A. Baronnet, CRMCC, Cnrs, Campus Luminy, Case 913,

13288 Marseilles, Cedex 9, France

16-20 International Conference on Ion Beam Modification of Materials
Ithaca, NY

J. W. Mayer, IBMM '84, Bard Hall, Cornell Univ., Ithaca, NY 14853

\section{AUGUST 1984}

1-3 The Physics of VLSI

Palo Alto, CA

K. Saraswat, McCullough 118, Stanford Univ., Stanford, CA 94305

(415) $497-2956$

$1-4$

Topical Conference on Optical Effects in Amorphous

Semiconductors

Salt Lake City, UT

W. W. Havens, Jr., 335 E 45 St., New York, NY 10017

(212) 661-9404

4-14 27th International Geological Congress

Moscow, USSR

Sec., 27th Int. Geological Cong., Inst. of Lithosphere,

Academy of Sciences, 22 Staromonetry, Moscow 109180, USSR

6-10 International Conference on the Physics of Semiconductors San Francisco, CA

R. Z. Bachrach, Xerox Palo Alto Res. Ctr., 3333 Coyote Hill

Road,

Palo Alto, CA 94304

12-17 Meeting of the Electron Microscopy Society of America Detroit, Ml

C. E. Lyman, Central Research \& Development Dept.

E. I. DuPont, Experimental Sta., Wlimington, DE 19898

(302) 772-4887

13-16 Ist International Conference on the Structure of Surfaces Berkeley, CA

M. A. VanHove, Dept. of Chem., Univ. of CA, Berkeley, CA 94720

13-16 International Conference on Superlattices, Microstructures and Microdevices

Champaign-Urbana, IL

J. D. Dow, Dept. of Phys., Univ, of Notre Dame, Notre Dame,

IN 46556

(219) 239-6407

6th International Conference on Thin Films

Stockholm, Sweden

S. Berg, ICTF-6, Inst. of Tech., Uppsala Univ., Box 534.

75121 Uppsala, Sweden

13-17 International Conference on Luminescence

Madison, WI

W. H. Yen, Dept. of Physics, 1150 Univ. Av., Madison, WI 53706

13-18 8th European Conference on Electron Microscopy Budapest, Hungary

Congress Bureau, MOTESZ, Budapest, POB32, H-1361, Budapest, Hungary

15-17 International Conference on Progress in Optical Physics ICO-Australian Acad. of Science

Melbourne, Australia

I. J. Wilson, CSIRO Div. of Chem. Phys., PO Box 160,

Clayton, Vic., Australia 3168

15-22 17th International Conference on Low Temperature Physics Karlsruhe, FRG

H. Hinsch, LT-17 Conf. Sec., Kernforschungszentrum,

Postfach 3640, 7500 Karlsruhe, FRG

19-22 AIChE Summer National Meeting

Philadelphia, PA 
AIChE, Meetings Dept., 345 E. 47 St., New York, NY 10017 (212) $705-7320$

20-24 International Conference on Defects in Insulating Crystals Salt Lake City, UT

F. Luty, Dept. of Phys., Univ. of Utah, Salt Lake City, UT 84112

(801) $581-7446$

26-29 International Topical Meeting on Fuel Reprocessing and Waste Management

Jackson Hole, WY

W. McClure, PO Box 3807, Idaho Falls, ID 83401

26-31 188th National Meeting of the American Chemical Society Philadelphia, PA

American Chem. Soc., 1155 16th St., NW, Washington, DC 20036

27-31 3rd International Conference on Solid Films and Surfaces Sydney, Australia

D. J. Miller, Sch. of Phys., Univ. of New South Wales, POB 1, Kensington, NSW 2033 Australia

27-31 6th General Conference of the European Physical Society Prague, Czechoslovakia

Phys. Sec., Union of Czech. Mathematicians \& Physicists,

Na Slovance 2, 182 00, Prague 8, Czech.

29-31 3rd International Symposium on Physical Metallurgy

of Cast Iron

Stockholm, Sweden

\section{SEPTEMBER 1984}

3-7 5th International Conference on Rapidly Quenched Metals Wurzburg, FRG

RQ 5, Postfach 2253, D-6450 Hanau, FRG

10-13 8th International Symposium on Chemical Reaction

Engineering

Edinburgh, Scotland

Dept. ISCRE, Conference Section, The Institution of Chemical

Engineers, 165-171 Railway Terrace, Rugby, Warwickshire

CV21 3HQ, England

13-14 Kinetics and Mass Transport of Silicate \& Oxide Systems London, UK

R. Freer, Dept. Electrical \& Electronic Eng.,

North Staffordshire Polytech., Beaconside, Stafford ST18 OAD

17-21 9th European Conference on Thermophysical Properties Manchester, UK

Mtg. Officer, IOP, 47 Belgrave Sq., London SW1X 8QX, UK

17-21 7th International Conference on Textures of Materials The Netherlands

Conf. Sec., PO Box 390, NL 3330 AJ Zwijndrecht,

The Netherlands

23-28 International Conference on the Science of Hard Materials Rhodes, Greece

25-28 Energy Pulse Modification of Semiconductors \& Related

Materials

Dresden, GDR

H. U. Jager, Centralinstitut für Dernforschung, Rossendorf,

8051 Dresden, Postfach 19, GDR

\section{OCTOBER 1984}

9-11 Conference on Advances in Materials Technology for the Process Industries

Atlanta, GA

Dale Miller, Deputy Executive Dir. for Public Affairs,

Natl. Assoc. of Corrosion Engineers, PO Box 218340,

Houston, TX 77218

9-12 37th Gaseous Electronics Conference

Boulder, $\mathrm{CO}$

W. W. Havens, Jr., 335 E. 45 St., New York, NY 10017

(212) 661-9404

21-26 5th International Meeting on Radiation Processing

San Diego, CA

J. Silverman, PO Box 3060, Woburn, MA 01888

29-2 Optical Society of America Annual Meeting

San Diego, Ca

Optical Society of America, 1816 Jefferson PI., NW,

Washington, DC 20036

31-2 4th International Conference on Ferrites

San Francisco, CA

\section{NOVEMBER 1984}

12-14 8th Conference on the Application of Accelerators in Research and Industry

Denton, TX

J. L. Duggan

Physics Dept., North Texas State Univ., NT Box 5368,

Denton, TX 76203

(817) $565-3252$

25-30 AIChE Annual Meeting

San Francisco, CA

AIChE, Meetings, Dept., 345 E. 47 St., New York, NY 10017

(212) $705-7320$

26-29 Materials Research Society Annual Meeting

Boston, MA

\section{DECEMBER 1984}

4-7 31st National Vacuum Symposium

Reno, NV

N. Hammond, 335 E. 45 St., New York, NY 10017

\section{JANUARY 1985}

20-24 American Physical Society/AAPT General Meeting Toronto, ONT

MARCH 1985

24-28 AIChE Spring National Meeting

Houston, TX

AIChE, Meetings Dept., 345 E. 47th St., New York, NY 10017 (212) $705-7320$

25-29 American Physical Society General Meeting

Baltimore, MD

W. Havens, Jr., 335 E. 45 St., New York, NY 10017 